PROCEEDINGS OF THE

AMERICAN MATHEMATICAL SOCIETY

Volume 140, Number 10, October 2012, Pages 3629-3643

S 0002-9939(2012)11209-2

Article electronically published on February 27, 2012

\title{
COMMUTATORS IN GROUPS DEFINABLE IN O-MINIMAL STRUCTURES
}

\author{
ELÍAS BARO, ERIC JALIGOT, AND MARGARITA OTERO
}

(Communicated by Julia Knight)

\begin{abstract}
We prove the definability and actually the finiteness of the commutator width of many commutator subgroups in groups definable in o-minimal structures. This applies in particular to derived series and to lower central series of solvable groups. Along the way, we prove some generalities on groups with the descending chain condition on definable subgroups and/or with a definable and additive dimension.
\end{abstract}

\section{INTRODUCTION}

Groups definable in o-minimal structures and groups of finite Morley rank share many properties. They are both equipped with a definable and additive finite dimension, satisfy the descending chain condition on definable subgroups, and have definably connected components, i.e., smallest definable subgroups of finite index. These formal properties suffice for many developments in the theory of groups of finite Morley rank, in which commutator subgroups are also very often definable by a well-known application of Zilber's stabilizer argument. For groups definable in o-minimal structures, even derived subgroups need not be definable. Using recent results from [6] on central extensions in that case, Annalisa Conversano exhibits in [3, Example 3.1.7] a definably connected group $G$ definable in an o-minimal expansion of the reals with $G^{\prime}$ not definable. Furthermore, $G$ is a central extension, by an infinite center isomorphic to $\langle[0,1),+(\bmod 1)\rangle$, of the simple group $\operatorname{PSL}_{2}(\mathbb{R})$.

In the present paper we will essentially prove that central extensions of this type are the only obstructions to the definability of commutator subgroups in the o-minimal case. In order to state our main theorem we need the following definition.

Definition 1.1. We say that a definably connected group $G$ definable in an ominimal structure is a strict central extension of a definably simple group if $Z(G)$ is infinite and $G / Z(G)$ is infinite nonabelian and definably simple.

We define a section of a group $G$ to be any quotient $H / K$ where $K \unlhd H \leq G$, and we speak of a definable section when both $K$ and $H$ are definable. In view of the previous example of a group definable in an o-minimal structure and with a

Received by the editors February 23, 2011 and, in revised form, April 9, 2011 and April 16, 2011.

2010 Mathematics Subject Classification. Primary 03C64; Secondary 20F12, 20F38, 20A15, $03 \mathrm{C} 60$.

Key words and phrases. Commutators, o-minimality, semi-algebraic groups, Lie groups.

The three authors are partially supported by MTM2011-22435. The first and third authors are partially supported by Grupos UCM 910444/GR35/10-A.

(C)2012 American Mathematical Society Reverts to public domain 28 years from publication 
nondefinable derived subgroup, we will mostly work with the following assumption. It consists merely in excluding "bad" sections of this type.

Definition 1.2. We say that a group $G$ definable in an o-minimal structure satisfies the assumption $(*)$ whenever the derived subgroup $(H / K)^{\prime}$ is definable for every definable section $H / K$ of $G$ that is a strict central extension of a definably simple group.

Theorem 1.3. Let $G$ be a group definable in an o-minimal structure, and $A$ and $B$ be two definable subgroups which normalize each other and such that $A^{\circ} B^{\circ}$ satisfies the assumption $(*)$. Then the subgroup $[A, B]$ is definable and $[A, B]^{\circ}=$ $\left[A^{\circ}, B\right]\left[A, B^{\circ}\right]$. Furthermore, any element of $[A, B]^{\circ}$ can be expressed as the product of at most $\operatorname{dim}\left([A, B]^{\circ}\right)$ commutators from $\left[A^{\circ}, B\right]$ or $\left[A, B^{\circ}\right]$ whenever $A^{\circ}$ or $B^{\circ}$ is solvable.

Theorem 1.3 implies that if $G$ is a group definable in an o-minimal structure with $G^{\circ}$ solvable, then the lower central series and derived series of $G$ consist of definable subgroups (see Corollary 6.8 below). In view of Conversano's example, on the other hand, the assumption $(*)$ is necessary if one wants a statement for definability as general as in Theorem 1.3

A commutator group $[A, B]$ as in Theorem 1.3 is the countable union of the definable sets $[A, B]_{n}$, consisting of the products of at most $n$ commutators $[a, b]$, or their inverses $[a, b]^{-1}$, with $(a, b)$ in $A \times B$. Our proof of Theorem 1.3 will consist of showing that $[A, B]$ has finite commutator width, i.e., that $[A, B]=[A, B]_{n}$ for some $n$. We emphasize that the existence of such a finite $n$ did not seem to be known even in the more classical case of semi-algebraic groups over real closed fields. Our argument for the proof of Theorem 1.3 will consist mainly in reducing to the case in which the relevant commutator subgroup is abelian, which is dealt with in Corollary 4.3 below, and we will always keep the best control on the commutator width.

We refer to [16] for a general introduction to o-minimal structures and to 12 for groups definable in o-minimal structures. We will frequently point out an analogy to the finite Morley rank case in [2]. All model-theoretic notions used here are rather elementary and can be found in any introductory book on model theory. We simply recall that an o-minimal structure is a first-order structure $\mathcal{M}$ with a total, dense, and without end-points definable order and such that every definable subset of $M$ is a Boolean combination of intervals with end-points in $M \cup\{ \pm \infty\}$.

Before passing to the proofs, we wish to comment on what our result says about Lie groups. Firstly note that a wide class of Lie groups, including compact Lie groups and the connected component of the identity of any real algebraic group, consists of semialgebraic groups, and in particular of definable groups. More generally, the fact that the real exponential field (as well as other expansions of the reals) is o-minimal [19] provides a larger class of Lie groups, in particular of nonsemialgebraic ones, which are definable in an o-minimal structure. In general, there exist Lie groups whose derived subgroup is not a Lie subgroup; see [11, Exercise 1.4.4] for an example of a solvable Lie group whose derived subgroup is only a virtual Lie subgroup. However, Theorem 1.3 shows that any solvable Lie group which is definable in an o-minimal structure has a definable derived subgroup; since definable subgroups are closed [12, Corollary 2.4] the derived subgroup must be a Lie subgroup. Hence, Theorem 1.3 provides already new examples of Lie groups 
whose derived subgroup is a Lie subgroup. More results of this sort are obtained for the commutator subgroups of a definable Lie group to which the theorem applies. Theorem 1.3 also provides new bounds on the commutator width (see comments after Corollary 6.8 and Question 7.2 below).

\section{Prelude to commutators}

We fix the following notation. If $a$ and $b$ are two elements of a group $G$, then we let $a^{b}=b^{-1} a b$ and $[a, b]=a^{-1} a^{b}$. If $A$ and $B$ are two subsets of $G$, then $[A, B]_{n}$ denotes, for $n \geq 1$, the set of products of at most $n$ elements of the form $[a, b]$ or $[a, b]^{-1}$, with $(a, b)$ in $A \times B$. We denote by $[A, B]$ the group generated by all commutators $[a, b]$. The commutator width, also frequently called the commutator length, of $[A, B]$ is the smallest $n$ such that $[A, B]=[A, B]_{n}$ if such an $n$ exists, and infinite otherwise. Since our proofs by induction will use passages to quotients, we will frequently "lift" commutators. This simply means that if $N$ is a normal subgroup of $G$ and $a, b \in G$, then $[a N, b N]=[a, b] N$ in $G / N$. In order to keep track of the commutator width when passing to quotients, the following elementary lemma will be crucial.

Lemma 2.1. Let $G$ be a group, $N$ a normal subgroup of $G$, and $A$ and $B$ two subsets of $G$ such that, modulo $N,[A, B]=[A, B]_{k}$ for some $k$. Suppose also that $[A, B] \cap N=[A, B]_{s} \cap N$ for some s. Then $[A, B]=[A, B]_{k+s}$.

Lemma 2.2. Let $A$ and $B$ be two subgroups of a group, with $A$ normalizing $B$. Suppose $C_{A}(B)$ is of finite index in $A$ and $C_{B}(A)$ is of finite index in $B$. Then $[A, B]$ is a finite subgroup of $B$.

Proof. By a result of Baer [15], it suffices to prove that $\{[a, b] \mid(a, b) \in A \times B\}$ is finite. Let $a_{i}, 1 \leq i \leq n$, be a set of representatives in $A$ of $A / C_{A}(B)$. Taking $a \in A$ and $b \in B$, with $a=a_{i} \alpha$ for some $i$ and some $\alpha$ in $C_{A}(B)$, then $[a, b]=$ $\left[a_{i}, b\right]^{\alpha}=\left[a_{i}, b\right]$ since $\alpha$ centralizes $B$ and $A$ normalizes $B$. Hence $[a, b]$ varies in $a_{i}^{-1} a_{i}{ }^{B}$, which is finite as $C_{B}\left(a_{i}\right)\left(\geq C_{B}(A)\right)$ has finite index in $B$.

We recall two classical formulas which are valid for any elements $a, b$, and $c$ in a group $G:[a, b c]=[a, c][a, b]^{c}$ and $[a b, c]=[a, c]^{b}[b, c]$. Therefore, if $A$ and $B$ are two subgroups of a group $G$, then the subgroup $[A, B]$ is always normalized by $A$ and $B$. Moreover, we easily get the following.

Fact 2.3. Let $G$ be a group, $x$ an element of $G$, and $H$ a subgroup such that $\{[h, x] \mid h \in H\} \subseteq C_{G}(H)$. Then the map ad $x: h \mapsto[h, x]$, from $H$ to $G$, is a group homomorphism, with $C_{H}(x)$ as kernel.

For any group $G$ we let $G^{1}=G^{(1)}=G^{\prime}=[G, G]$, the derived subgroup of $G$, and we define by induction on $n \geq 1$ the lower central series and derived series as follows: $G^{n+1}=\left[G, G^{n}\right]$ and $G^{(n+1)}=\left[G^{(n)}, G^{(n)}\right]$. We say that $G$ is n-nilpotent (resp. $n$-solvable) whenever $G^{n}=1$ (resp. $G^{(n)}=1$ ). We also define the upper central series as follows: $Z_{1}(G)=Z(G)$ is the center of $G$, and by induction $Z_{n+1}(G)$ is the preimage in $G$ of $Z\left(G / Z_{n}(G)\right)$. Our inductive proofs will naturally use the following elementary fact (see, e.g., [17, 0.1.7-8]).

Fact 2.4. Let $n \geq 1$.

(a) If $G$ is a nilpotent group of class $n$, then $G^{i} \leq Z_{n-i}(G)$, for $0 \leq i \leq n$.

(b) A group $G$ is nilpotent of class $n$ if and only if $Z_{n}(G)=G$ and $Z_{n-1}(G)<G$. In this case $G / Z(G)$ is nilpotent of class $n-1$. 


\section{Groups with $d c c$}

Throughout the present section, $G$ is a group interpretable in a structure $\mathcal{M}$, and definability may refer to $\mathcal{M}^{\text {eq }}$. We work under the mere assumption that $G$ satisfies the descending chain condition on definable subgroups (dcc for short): any strictly descending chain of definable subgroups of $G$ is finite. Groups definable in an o-minimal structure $\mathcal{M}$ satisfy the $d c c$ [12, Corollary 2.4], and thus we can freely apply the results of the present section to such groups. Any definable group with no proper definable subgroup of finite index is said to be definably connected. We start with two general facts about definably connected groups (independent of the $d c c)$.

Fact 3.1. Let $G$ be a definably connected group.

(a) Any definable action of $G$ on a finite set is trivial.

(b) If $G^{\prime}$ is finite, then $G$ is abelian.

(c) If $Z(G)$ is finite, then $G / Z(G)$ is centerless.

Proof. (a) The fixator of any element, being of finite index, must be $G$. (b) For any $g$ in $G, g^{G} \in g G^{\prime}$, which is finite, and since $G$ acts definably on $g^{G}$ by conjugation, claim $(a)$ shows that $g$ is in $Z(G)$. (c) See [2, Lemma 6.1].

Fact 3.2. Let $A$ and $B$ be two definably connected definable subgroups of a definable group $G$, with $A$ normalizing $B$. Then $A B$ is a definably connected definable subgroup of $G$.

Proof. If $H$ is a definable subgroup of finite index of $A B$, then $H \cap A$ has finite index in $A$ and $A \leq H$; similarly, $B \leq H$, and $A B=H$.

If $H$ is a definable subgroup of a group with the $d c c$, then $H$ has a smallest definable subgroup of finite index, the intersection of all of them. It is denoted by $H^{\circ}$ and called the definably connected component of $H$. Clearly $H^{\circ}$ is definably characteristic in $H$, and in particular normal in $H$. Any subset $X$ of a group $G$ with the $d c c$ is contained in a smallest definable subgroup, the intersection of all of them, called the definable hull of $X$ and denoted by $H(X)$.

Remark 3.3. Let $G$ be a definable group with the $d c c$ and let $X$ and $K$ be two subsets of $G$ with $X K$-invariant. Then $H(X)$ is $K$-invariant.

Proof. For any $k$ in $K, X^{k}=X$ and hence $X \subseteq H(X) \cap H(X)^{k}$. So $H(X)=$ $H(X) \cap H(X)^{k}$, and thus $H(X) \leq H(X)^{k}$. But if $H(X)<H(X)^{k}$, then $X \subseteq$ $H(X)^{k^{-1}}<H(X)$, a contradiction.

Fact 3.4. Let $G$ be a group with the $d c c$. If $X$ and $Y$ are two subgroups of $G$, then $[H(X), H(Y)] \leq H([X, Y])$. In particular if $X$ is $n$-nilpotent (resp. $n$-solvable), then $H(X)$ is $n$-nilpotent (resp. $n$-solvable) as well.

Proof. This is proven as in [2, Lemma 5.37], using Remark 3.3 instead of [2, Lemma 5.35].

If $G$ is any group, then $F(G)$ denotes the subgroup generated by all normal nilpotent subgroups of $G$ and is called the Fitting subgroup of $G$. It is clearly a normal subgroup of $G$.

Fact 3.5. Let $G$ be a group with the $d c c$. Then $F(G)$ is nilpotent and definable. 
Proof. The group $F(G)$ is nilpotent by [17, Theorem 1.2.11]. By Remark 3.3 and Fact 3.4 $H(F(G))$ is normal and nilpotent, so it must coincide with $F(G)$.

Lemma 3.6. Let $G$ be a nilpotent group with the dcc and $H$ an infinite normal subgroup of $G$. Then $H \cap Z(G)$ is infinite.

Proof. As in [2, Ex. 5, p. 98], we proceed by induction on the nilpotency class of $G$. We may assume that our counterexample of a minimal nilpotency class is nonabelian. So suppose $H \cap Z(G)$ is finite. Applying the induction hypothesis in $G / Z(G)$, which has smaller nilpotency class by Fact 2.4, we see that $H Z(G) / Z(G) \cap$ $Z(G / Z(G))$ is infinite. Replacing $H$ by $H \cap Z_{2}(G)$, we may thus assume that $H \leq Z_{2}(G)$. Let $x \in G$. Then the map ad ${ }_{x}: H \rightarrow G: h \mapsto[h, x]$ takes its values in $H \cap Z(G)$. By Fact 2.3 this is a group homomorphism, with $C_{H}(x)$ as its kernel. Hence the latter must have finite index in $H$. Besides, $Z(G)=C_{G}\left(x_{1}, \ldots, x_{n}\right)$ for some $x_{1}, \ldots, x_{n} \in G$ by $d c c$. In particular $H \cap Z(G)=C_{H}\left(x_{1}\right) \cap \cdots \cap C_{H}\left(x_{n}\right)$ has finite index in $H$. But since $H$ is infinite and $H \cap Z(G)$ is finite, this is a contradiction.

We now pass to a finer analysis of abelian and nilpotent groups.

Fact 3.7. Let $A$ be an abelian group with the $d c c$. Then:

(a) $A=D \oplus B$ for some definable divisible subgroup $D$ and some (interpretable but not necessarily definable) complement $B$ of bounded exponent.

(b) $A=D C$ for some definable and characteristic subgroups $D$ and $C$, with $D$ divisible as above and $C$ of bounded exponent. Furthermore $D \cap C$ is finite whenever $A$ contains no infinite elementary abelian $p$-subgroup.

Proof. This is proven as in [2, Theorem 6.7], originally in [9].

Corollary 3.8. Let $A$ be a definably connected abelian group with the dcc and with no infinite elementary abelian p-subgroup. Then $A$ is divisible.

Proof. Let $A=D C$ be a decomposition of $A$ as in Fact $3.7(b)$, with $D$ divisible and $C$ of bounded exponent. For $p$ any prime and any $n \geq 0$, let $C_{p^{n}}=$ $\left\{g \in C \mid g^{p^{n}}=1\right\}$. Since $C$ has bounded exponent, the $p$-primary component of $C$ has the form $C_{p^{N}}$ for some $N$, and it is in particular definable. By assumption, $C_{p}$ is finite. Considering the group homomorphism $x \longmapsto x^{p}$, we see by induction on $n$ that each subgroup $C_{p^{n}}$ is finite. In particular, the $p$-primary component $C_{p^{N}}$ of $C$ is finite. But, as $C$ has bounded exponent, there are only finitely many such $p$-primary components. Hence $C$ is finite, and $A=D$.

Fact 3.9. Let $G$ be a group with the $d c c, N$ a normal definable subgroup of $G$, and $x$ an element of $G$ such that $x$ has finite order $n$ modulo $N$. Then the coset $x N$ contains an element of finite order, having the same prime divisors as $n$.

Proof. Replacing $G$ by $H(x)$, we may assume $G$ abelian by Fact 3.4 . Now decomposing $G$ as in Fact 3.7 one can argue as in [2, Ex. 11, p. 93].

Lemma 3.10. Let $G$ be a nilpotent group with the dcc and with $G^{\circ}$ divisible. Let Tor $(G)$ denote the set of torsion elements of $G$. Then:

$(a) \operatorname{Tor}(G)$ is a subgroup of $G$, and it is the direct product of its Sylow psubgroups. Furthermore it commutes with $G^{\circ}$.

$\left(a^{\prime}\right)$ Tor $\left(G^{\circ}\right)$ is in $Z^{\circ}\left(G^{\circ}\right)$, and in particular it is a divisible abelian subgroup of $G^{\circ}$. Besides, any definable section of $G^{\circ} / Z^{\circ}\left(G^{\circ}\right)$ is torsion-free. 
(b) $G=G^{\circ} * B$ (central product) for some finite subgroup $B$ of Tor $(G)$.

(c) If $G^{\circ}$ has no infinite elementary abelian p-subgroup, then the subgroup $B$ as in claim (b) may be chosen to be a finite characteristic subgroup.

Proof. (a) The fact that Tor $(G)$ is a subgroup and the direct product of its Sylow $p$-subgroups is true in any nilpotent group $([14,5.2 .7])$. Since $G^{\circ}$ is $p$-divisible for any prime $p$, it commutes with any Sylow $p$-subgroup by [18, $4.2 ; 4.7 ; 6.13]$. In particular Tor $(G)$ commutes with $G^{\circ}$.

$\left(a^{\prime}\right)$ By $(a)$, Tor $\left(G^{\circ}\right) \leq Z\left(G^{\circ}\right)$. By divisibility of $G^{\circ}$, Tor $\left(G^{\circ}\right)$ is also a divisible (abelian) subgroup of $G^{\circ}$. In particular any finite quotient of the subgroup Tor $\left(G^{\circ}\right)$ must be trivial. Hence Tor $\left(G^{\circ}\right) Z^{\circ}\left(G^{\circ}\right) / Z^{\circ}\left(G^{\circ}\right)$ is trivial, and Tor $\left(G^{\circ}\right) \leq Z^{\circ}\left(G^{\circ}\right)$. Our last claim follows then from the lifting of torsion given by Fact 3.9

(b) The subgroup Tor $(G)$, being a torsion solvable group, is locally finite [7, Lemma 1.A.2, p.2]. Pick up finitely many representatives of all cosets of $G^{\circ}$ in $G$ which, by Fact 3.9, can be taken in Tor $(G)$; the subgroup $B$ they generate is finite. Since $G^{\circ}$ commutes with $\operatorname{Tor}(G)$ by $(a), G=G^{\circ} * B$.

(c) Let $n$ be the order of $B$ and $\tilde{B}=\left\{g \in \operatorname{Tor}(G) \mid g^{n}=1\right\}$. Let $g \in \tilde{B}$. Then $g=b h$ for some $b \in B$ and $h \in G^{\circ}$. Since $[b, h]=1$, we see that $1=g^{n}=h^{n}$. Now, the torsion subgroup of $G^{\circ}$ consists of direct products of quasicyclic Prüfer $p$-groups. Our assumption on $G^{\circ}$ implies that for each $p$ the direct product must have finite width. In particular, there are only finitely many elements $h$ in $G^{\circ}$ satisfying $h^{n}=1$. Hence $\tilde{B}$ is finite. Therefore, as in $(b),\langle\tilde{B}\rangle$ is finite, and it is clearly characteristic in $G$. So we may replace $B$ by $\langle\tilde{B}\rangle$.

The following successive corollaries generalize [4, Lemma 2.14] from the finite Morley rank case.

Corollary 3.11. Let $G$ be a definably connected group with the dcc. Let $H$ be a definable normal nilpotent subgroup of $G$, with $H^{\circ}$ divisible and without infinite elementary abelian p-subgroups. Then $H=(Z(G) \cap H) H^{\circ}$, and Tor $(H) \leq Z(G)$.

Proof. The first point follows from Lemma $3.10(c)$ and Fact $3.1(a)$. For the second one, we have seen in the proof of Lemma 3.10 (c) that the set of elements of $H^{\circ}$ of order dividing $n$ is finite, for every $n$, and thus Fact 3.1 (a) also gives that the torsion of $H^{\circ}$ is central in $G$. Now the full torsion subgroup Tor $(H)$ of $H$ is in $Z(G)$ by Lemma $3.10(c)$ and Fact $3.1(a)$ as well.

Corollary 3.12. Let $G$ be a definably connected group with the dcc. Suppose that $F^{\circ}(G)$, which is definable and nilpotent by Fact 3.5, is divisible and without infinite elementary abelian p-subgroups. Then $F(G)=Z(G) F^{\circ}(G)$ and $\operatorname{Tor}(F(G)) \leq$ $Z(G)$.

We conclude this section with a characterization of central extensions. We will freely apply it to groups definable in o-minimal structures, since their abelian subgroups of bounded exponent are always finite [12, Theorem 5.1].

Lemma 3.13. Let $G$ be a definably connected group with the dcc, and let $H$ be a definable normal subgroup such that $H^{\circ}$ contains no infinite elementary abelian p-subgroups. Then $H \leq Z(G)$ if and only if $H^{\circ} \leq Z(G)$.

Proof. Suppose $H^{\circ} \leq Z(G)$. Fact 3.1 (a) implies that $H / H^{\circ} \leq Z\left(G / H^{\circ}\right)$. In particular $H^{\prime} \leq H^{\circ}(\leq Z(H))$ and so $H$ is nilpotent. Besides, $H^{\circ}$ is divisible by Corollary 3.8. Now by Corollary 3.11 we get $H \leq Z(G)$. 


\section{Groups with a Definable and additive Dimension}

Throughout the present section, $G$ is a group interpretable in a structure $\mathcal{M}$, and definability refers to $\mathcal{M}^{\mathrm{eq}}$. We suppose that to each definable set in Cartesian powers of $G$ is attached a dimension in $\mathbb{N}$, denoted by $\operatorname{dim}$ and satisfying the following axioms.

(A1) (Definability) If $f$ is a definable function between two definable sets $A$ and $B$, then for every $m$ in $\mathbb{N}$ the set $\left\{b \in B \mid \operatorname{dim}\left(f^{-1}(b)\right)=m\right\}$ is a definable subset of $B$.

(A2) (Additivity) If $f$ is a definable function between two definable sets $A$ and $B$, whose fibers have constant dimension $m$ in $\mathbb{N}$, then $\operatorname{dim}(A)=\operatorname{dim}(f(A))+m$.

(A3) (Finite sets) A definable set $A$ is finite if and only if $\operatorname{dim}(A)=0$.

Axioms (A2) and (A3) guarantee that if $f$ is a definable bijection between two definable sets $A$ and $B$, then $\operatorname{dim}(A)=\operatorname{dim}(B)$. Using axiom (A2), one sees that if $K \leq H \leq G$ are definable subgroups of $G$, then $\operatorname{dim}(H)=\operatorname{dim}(K)+\operatorname{dim}(H / K)$. Notice that groups definable in o-minimal structures are equipped with a dimension satisfying axioms (A1)-(A3) (see [16, Chapter 4]).

Lemma 4.1. Let $G$ be a group equipped with a dimension satisfying axioms (A1)(A3). If $A$ and $B$ are two definable subgroups of $G$, then $\operatorname{dim}(A B)=\operatorname{dim}(A)+$ $\operatorname{dim}(B)-\operatorname{dim}(A \cap B)$.

Proof. It suffices to consider the definable map $(a, b) \longmapsto a b$ from $A \times B$ onto $A B$. Its fibers are in definable bijection with $A \cap B$.

The next lemma can be seen, in this general context, as a very rudimentary version of Zilber's generation lemma ([2, Theorem 5.26]). Its corollary is going to be the basic tool for the proof of our main theorem.

Lemma 4.2. Let $G$ be a group equipped with a dimension satisfying axioms (A1)(A3), and let $A_{i}, i \in I$, be a family of definably connected definable subgroups of $G$ which pairwise normalize each other. Then $H:=\left\langle A_{i} \mid i \in I\right\rangle=A_{i_{1}} \cdots A_{i_{k}}$ for finitely many $i_{1}, \ldots, i_{k} \in I$, and in particular $H$ is definable. Furthermore we can take $k \leq \operatorname{dim} H$.

Proof. Any finite product $A_{i_{1}} \cdots A_{i_{k}}$ is a definably connected and definable subgroup by Fact 3.2 Now one sees with Lemma 4.1 that any such finite product $A_{i_{1}} \cdots A_{i_{k}}$ of maximal dimension is equal to $H$. Again by Lemma 4.1, we may choose $k \leq \operatorname{dim} H$.

It is a question whether one can relax the normalization hypothesis in Lemma 4.2 , assuming that the ambient group, say, is nilpotent.

Corollary 4.3. Let $G$ be a group equipped with a dimension satisfying axioms (A1)-(A3), $H$ a definably connected definable subgroup, and $X$ a subset of $G$. Suppose that $[H, X]$ is abelian and centralized by $H$. Then $[H, X]$ is a definably connected definable subgroup and $[H, X]=[H, X]_{\operatorname{dim}([H, X])}$.

Proof. Let $x$ be in $X$. Then the map ad $x: H \rightarrow G$ is a group homomorphism by Fact $[2.3$ and clearly it is definable. Hence its image $[H, x]$ is a definably connected definable subgroup of $G$. Since $[H, X]$ is generated by these subgroups $[H, x]$, we conclude by applying Lemma 4.2 . 
If $G$ is any group, $R(G)$ denotes the subgroup generated by all normal solvable subgroups of $G$ and is called the solvable radical of $G$. We next prove the definability and the solvability of the solvable radical in our rather abstract context. Before that, we observe the following consequence of Fact $3.1(a)$.

Remark 4.4. Let $G$ be any definably connected group. If $R(G)$ is finite, then $R(G)=Z(G)$ and $G / R(G)$ has a trivial solvable radical.

Lemma 4.5. Let $G$ be a group equipped with a dimension satisfying axioms (A1)(A3) and with the dcc. Then $R(G)$ is definable and solvable.

Proof. By Remark 3.3 and Fact 3.4, $R(G)$ is generated by the set of definable normal solvable subgroups of $G$, and it suffices to show it is solvable. Let $K$ denote the maximal definably connected definable normal solvable subgroup of $G$, which exists by Lemma 4.2 (and which, of course, is going to be $R^{\circ}(G)$ ).

Any definable normal solvable subgroup $N$ of $G$ has a finite image in $G / K$. So replacing $G$ by $G / K$ we may assume that all normal solvable subgroups are finite. If $N$ is such a subgroup, then $N \leq C_{G}\left(G^{\circ}\right)$ by Fact $3.1(a)$. But $C_{G}\left(G^{\circ}\right)$ is a finite subgroup of $G$, as otherwise $C_{G}^{\circ}\left(G^{\circ}\right)$ is a nontrivial definably connected definable subgroup of $G$, hence in $G^{\circ}$, and then in the center of $G^{\circ}$, which is finite, a contradiction. Hence any normal solvable subgroup of $G$ is in the finite subgroup $C_{G}\left(G^{\circ}\right)$ of $G$, and in particular in $R\left(C_{G}\left(G^{\circ}\right)\right)$, which is solvable.

We note, parenthetically, that in the literature on groups definable in o-minimal structures it is only $R^{\circ}(G)$ which has been defined so far.

If $G$ is a group as in Lemma 4.5, then $R(G)$ is a solvable definable subgroup, and $F(G)$ is a nilpotent definable subgroup by Fact 3.5. Of course, we have $F(G) \unlhd$ $R(G) \unlhd G$, and the same inclusions hold with definably connected components. If $G$ is definably connected and $R(G)$ is finite, then Remark 4.4 shows that $Z(G)=$ $F(G)=R(G)$ and $G / R(G)$ has no nontrivial normal abelian subgroup. In this case $G$ can have only finite normal abelian subgroups. The definition of semisimplicity for groups may vary in the literature, depending on whether the authors admit a finite center or not. Here we say that a definably connected group $G$ as in Lemma4.5 is semisimple whenever $R^{\circ}(G)=1$. By Remark 4.4, this is equivalent to requiring that $Z(G)=R(G)$ is finite and $G / R(G)$ has trivial abelian normal subgroups.

\section{Groups Definable in O-Minimal StruCtures}

The framework from now on is the following: $\mathcal{M}$ is an o-minimal structure and $G$ is a group definable in $\mathcal{M}$. Here we insist on definability, as opposed to interpretability. However, both concepts coincide provided the o-minimal structure expands an ordered group, due to elimination of imaginaries [16, Proposition 6.1.2]. Even if the o-minimal structure $\mathcal{M}$ itself may not eliminate imaginaries, in some sense $G$ does. In fact, $G$ has strong definable choice in the following sense.

Fact 5.1 ([5, Theorem 7.2]). Let $G$ be a group definable in an o-minimal structure $\mathcal{M}$, and let $\{T(x) \mid x \in X\}$ be a definable family of nonempty definable subsets of $G$. Then there is a definable function $t: X \longrightarrow G$ such that for all $x, y \in X$ we have $t(x) \in T(x)$ and if $T(x)=T(y)$, then $t(x)=t(y)$.

Therefore, when making proofs by induction below, we will freely pass to definable sections of groups definable in o-minimal structures and still consider them as definable. 
As already mentioned, a group $G$ definable in an o-minimal structure satisfies the $d c c$ and is equipped with a definable and additive dimension, so all the results of Sections 3 and 4 apply to such a $G$. In particular, $R(G)$ is definable and solvable. When $G$ is definably connected, $G / R^{\circ}(G)$ is semisimple and $G / R(G)$ has the following description following a solution of a version of the Cherlin-Zilber conjecture for groups in o-minimal structures [6, Fact 1.2(3)].

Fact 5.2. Let $G$ be an infinite definably connected group definable in an o-minimal structure, with $R(G)=1$. Then $G$ is a finite direct product of definably simple definable subgroups (i.e., nonabelian and with no proper nontrivial normal definable subgroups).

Fact 5.3. Let $G$ be a definably simple group definable in an o-minimal structure. Then $G=[G, G]_{k}$ for some $k$, and in particular $G=G^{\prime}$; i.e., $G$ is perfect.

Proof. We may suppose that $G$ is infinite and that the o-minimal structure is sufficiently saturated. If $G$ is not definably compact, then by [13, Corollary 6.3] $G$ is abstractly simple as a group, and $G=G^{\prime}$. Now $G$ is the countable union of the definable sets $[G, G]_{k}$, so by saturation $G=[G, G]_{k}$ for some $k$. Consider now the case that $G$ is definably compact. Then $G$ is definably isomorphic to a semialgebraic group over a real closed field $\mathcal{R}$ which is definable in our original ominimal structure (see [6. Fact 1.2(1)]). Hence we can see $G$ as a definably compact group definable over an o-minimal expansion of an ordered field. Then we can get $G^{\prime}=[G, G]_{k}$ for some $k$ as in the proof of $[\underline{6}$, Corollary $6.4(\mathrm{i})]$.

In the rest of the section we consider the structure of definably connected solvable groups definable in o-minimal structures. We first note that there is an o-minimal version of the Lie-Kolchin-Mal'cev theorem.

Fact 5.4 ([5, Theorem 6.9]). Let $G$ be a definably connected solvable group definable in an o-minimal structure. Then $G^{\prime}$ is nilpotent.

As in the case of finite Morley rank, the proof of Fact 5.4 is based ultimately on linearization. It is a question whether a version of Fact 5.4 can be proved under more general assumptions such as those used in Section 3 or 4 .

Proposition 5.5. Let $G$ be a definably connected solvable group definable in an o-minimal structure. Then $G^{\prime} \leq F^{\circ}(G)$. In particular $G / F^{\circ}(G)$ and $G / F(G)$ are divisible abelian groups.

Proof. Notice that both quotients $G / F^{\circ}(G)$ and $G / F(G)$ are definably connected as is $G$. By Fact 5.4. $G^{\prime} \leq F(G)$. It follows that $\left(G / F^{\circ}(G)\right)^{\prime}=G^{\prime} F^{\circ}(G) / F^{\circ}(G)$ is finite. Now, by Fact $3.1(b), G / F^{\circ}(G)$ is abelian. Hence $G^{\prime} \leq F^{\circ}(G)$ and $G / F(G)$ is also abelian. The divisibility of $G / F^{\circ}(G)$ and $G / F(G)$ is known to be true for any definably connected nilpotent group definable in an o-minimal structure by 5 , Theorem 6.10].

Corollary 5.6. Let $G$ be a nontrivial definably connected solvable group definable in an o-minimal structure. Then $F^{\circ}(G)$ is nontrivial. In particular, $G$ has an infinite abelian characteristic definable subgroup.

Proof. We may suppose $G$ to be nonabelian. Then $F^{\circ}(G)$ is infinite by Proposition [5.5] and Fact $3.1(b)$. For the last claim we can use the fact that $F(G)$ has an infinite center, which follows from Fact 3.5 and Lemma 3.6. 
The following lemma is a very preliminary version of our main theorem; it will indeed be used in a reduction from the solvable to the nilpotent case in its proof.

Lemma 5.7. Let $G$ be a nontrivial definably connected solvable group definable in an o-minimal structure. Then $G^{\prime} \leq H<G$ for some definably connected and definably characteristic proper subgroup $H$ definable in $G$.

Proof. We may assume $G$ to be nonabelian and, by Fact 3.5 and Proposition 5.5 , $F^{\circ}(G)=G$; i.e., $G$ is $n$-nilpotent for some $n \geq 2$. But now by Fact 2.4, $G=Z_{n}(G)$ and $Z_{n-1}(G)<G$, and we also have $G^{\prime} \leq Z_{n-1}(G)$. Clearly, all groups $Z_{i}(G)$ are definable. Now one sees as in the proof of Proposition 5.5 that $G / Z_{n-1}^{\circ}(G)$ has a finite derived subgroup, which must then be trivial by Fact $3.1(b)$. Hence $G^{\prime} \leq Z_{n-1}^{\circ}(G)<G$, and we may take $H=Z_{n-1}^{\circ}(G)$.

Corollary 5.8. Let $G$ be a group definable in an o-minimal structure, with $G^{\circ}$ solvable. Then $G$ satisfies the assumption (*) of Definition 1.2 .

Proof. By Lemma 5.7 $G^{\circ}$ cannot have nonabelian definably simple definable sections.

\section{MAIN THEOREM}

We first prove a version of Theorem 1.3 for $A$ and $B$ definably connected.

Theorem 6.1. Let $G$ be a group definable in an o-minimal structure, $A$ and $B$ be two definably connected definable subgroups which normalize each other and such that $A B$ satisfies the assumption $(*)$. Then $[A, B]$ is a definably connected definable subgroup, and moreover $[A, B]=[A, B]_{\operatorname{dim}([A, B])}$ whenever $A$ or $B$ is solvable.

Proof. Notice that $[A, B] \leq A \cap B$ since $A$ and $B$ normalize each other. We proceed by induction on

$$
d:=\min (\operatorname{dim}(A), \operatorname{dim}(B)) .
$$

The induction starts with the trivial case $d=0$. Assume from now on that $G$ is a potential counterexample to our statement, with $d \geq 1$ minimal. We start with a series of reductions. Notice that since $A B$ satisfies the assumption $(*)$, every definable section of $A B$ does also.

Claim 6.2. Let $C$ be a definable subgroup of $A \cap B$ normalized by $A$ and $B$ and with $\operatorname{dim}(C)<d$. If $C \not \leq Z(A) \cap Z(B)$, then $[A, B]$ is definable and definably connected, and $[A, B]=[A, B]_{\operatorname{dim}([A, B])}$ if $A$ or $B$ is solvable.

Proof. Suppose that $C$ does not centralize one of the two groups $A$ or $B$, say $C \not \leq Z(B)$. We now have $C^{\circ} \not \leq Z(B)$ by Lemma 3.13. In particular $\left[C^{\circ}, B\right]$ is nontrivial. Since $\operatorname{dim}(C)<d$, our inductive assumption implies that $\left[C^{\circ}, B\right]$ is definable and definably connected and that $\left[C^{\circ}, B\right]=\left[C^{\circ}, B\right]_{\operatorname{dim}\left(\left[C^{\circ}, B\right]\right)}$ whenever $A$ or $B$ is solvable (since in both cases $C^{\circ}$ is solvable). Notice that $\left[C^{\circ}, B\right]$ is normal in both $A$ and $B$. We now work in $N(U) / U$, where $U=\left[C^{\circ}, B\right]$, and denote by the notation "-" the quotients by $U$. Applying our inductive assumption in $N(U) / U$, we get that $[\bar{A}, \bar{B}]$ is definable and definably connected, and $[\bar{A}, \bar{B}]=[\bar{A}, \bar{B}]_{\operatorname{dim}([\bar{A}, \bar{B}])}$ if $A$ or $B$ is solvable.

But clearly $[\bar{A}, \bar{B}]=\overline{[A, B]}=[A, B] / U$ since $U \leq[A, B]$, and it follows that $[A, B]$ is definable and definably connected. By additivity of the dimension, we now get that $\operatorname{dim}([A, B])=\operatorname{dim}(\overline{[A, B]})+\operatorname{dim}(U)$. Hence, if $A$ or $B$ is solvable, we 
get also that $[A, B]=[A, B]_{\operatorname{dim}([A, B])}$ by the additivity of the commutator width provided by Lemma 2.1

Claim 6.3. We may assume that $A=A \cap B \not \leq Z(A) \cap Z(B)$.

Proof. If $(A \cap B) \leq Z(A) \cap Z(B)$, then in particular $[A, B] \leq Z(A) \cap Z(B)$ and Corollary 4.3 would give all the conclusions of Theorem $[6.1$ for $[A, B]$. Since we are dealing with a potential counterexample, we may thus assume $A \cap B \not$ $Z(A) \cap Z(B)$.

Now supposing $\operatorname{dim}(A \cap B)<d$, we may apply Claim 6.2 with $C=A \cap B$. Hence, we may also assume $\operatorname{dim}(A \cap B)=d$. Since $A$ and $B$ are definably connected it follows that $A \cap B$ equals $A$ or $B$, say $A$.

In particular we are now in the situation in which $A \leq B$.

Claim 6.4. We may assume $A=B$.

Proof. We have to show that if the conclusions of Theorem 6.1 are valid for $[A, A]=$ $A^{\prime}$, then they are also valid for $[A, B]$. So suppose $[A, A]$ is definable and definably connected, and $[A, A]=[A, A]_{\operatorname{dim}\left(A^{\prime}\right)}$ if $A$ is solvable.

Working modulo $A^{\prime}$ (which is normal in $B$ ), we may assume $A$ to be abelian. Then $[A, B] \leq A=Z(A)$, and Corollary 4.3 gives that $[A, B]=[A, B]_{\operatorname{dim}([A, B])}$ is definable and definably connected.

We can now come back to the original groups $A$ and $B$, i.e., not divided by $A^{\prime}$. Since $A^{\prime} \leq[A, B]$, we deduce as in the proof of Claim 6.2 the definability and definable connectedness of $[A, B]$. Since $A \leq B, A$ is solvable if $A$ or $B$ is. Hence we also deduce as in the proof of Claim 6.2, using the additivity of the dimension and of the commutator width given by Lemma 2.1, that $[A, B]=[A, B]_{\operatorname{dim}([A, B])}$ if $A$ or $B$ is solvable.

We are now in the situation $A=B$, and in view of Claim 6.2 we may suppose that any proper definable normal subgroup $C$ of $A$ is central in $A$. We split our final analysis into two cases.

Case $A$ solvable. We may suppose $A$ to be nonabelian. By Lemma $5.7, A^{\prime} \leq C$ for some proper normal definably connected definable subgroup $C$, and since we may assume $C \leq Z(A)$ we have $A^{\prime} \leq Z(A)$ (i.e., $A$ is 2-nilpotent). Now Corollary 4.3 (applied with $H=X=A$ ) gives all the desired conclusions again in this case, including the control of the commutator width: $[A, A]=[A, A]_{\operatorname{dim}\left(A^{\prime}\right)}$.

Case $A$ nonsolvable. Now suppose that $R(A)<A$. Notice that in this case we just want to conclude the definability and the definable connectedness of $A^{\prime}$ (here we do not address the commutator width). Notice also that we actually may suppose, by Claim 6.2. that $A$ is indecomposable in the following sense: it cannot be the product $R_{1} R_{2}$ of two proper definable normal subgroups $R_{1}$ and $R_{2}$. By the structure of semisimple groups definable in o-minimal structures, Fact [5.2 we get that $A / R(A)$ consists of a single definably simple factor. Notice also that the proper normal definable subgroup $C=R(A)$ is central in $A$, i.e., $R(A)=Z(A)$. To summarize, $A$ is a central extension of a nonabelian definably simple group $A / R(A)$.

By Fact 5.3. $A / Z(A)=[A / Z(A), A / Z(A)]_{k}$ for some $k$, and lifting commutators, one gets $A=[A, A]_{k} \cdot Z(A)$ and in particular $A=A^{\prime} \cdot Z(A)$. We distinguish two final subcases. 
Subcase $Z(A)$ finite. In this case we clearly have $[A, A] \cap Z(A)=[A, A]_{s} \cap Z(A)$ for some finite $s \geq 1$. Now $A^{\prime}=[A, A]=[A, A]_{k+s}$ by Lemma 2.1 and in particular $A^{\prime}$ is definable. Since $A=A^{\prime} Z(A)$ and $Z(A)$ is finite, $A^{\prime}$ has finite index in $A$. Now since $A$ is definably connected we conclude that $[A, A]=A^{\prime}=A$ is definable and definably connected, as desired.

Subcase $Z(A)$ infinite. In this final case $A$ is a strict central extension of a nonabelian definably simple group. Since our original group $A B$ satisfied the assumption $(*)$, the same is true for $A$ at this stage. Hence, the assumption $(*)$ now says that $A^{\prime}$ is a definable subgroup of $A$. Notice that $A^{\prime}$ cannot be finite, as otherwise $A$ is abelian by Fact $3.1(b)$. If $A^{\prime}<A$, then our general application of Claim 6.2 implies that we may assume $A^{\prime} \leq Z(A)$, which is excluded since $A$ is not solvable. There remains only the case in which $[A, A]=A^{\prime}=A$ is definable and definably connected, as desired.

This finishes the proof of Theorem 6.1 in all cases.

Corollary 6.5. Let $G$ be a group definable in an o-minimal structure, and let $A$ and $B$ be two definable subgroups which normalize each other with $A=A^{\circ}$ and such that $A B^{\circ}$ satisfies the assumption $(*)$. Then $[A, B]$ is a definably connected definable subgroup, and moreover $[A, B]=[A, B]_{\operatorname{dim}([A, B])}$ whenever $A$ or $B^{\circ}$ is solvable.

Proof. We have $[A, B]$ and $\left[A, B^{\circ}\right]$ normal in both $A$ and $B$. By Theorem 6.1 we have $\left[A, B^{\circ}\right]$ definable and definably connected, and $\left[A, B^{\circ}\right]=\left[A, B^{\circ}\right]_{\operatorname{dim}\left(\left[A, B^{\circ}\right]\right)}$ if $A$ or $B^{\circ}$ is solvable.

Working in $N\left(\left[A, B^{\circ}\right]\right) /\left[A, B^{\circ}\right]$ and denoting by the notation "-" the quotients by $\left[A, B^{\circ}\right]$, we may assume that $\left[\bar{A}, \overline{B^{\circ}}\right]=1$. Now the definably connected group $\bar{A}$ acts by conjugation on the finite quotient $\bar{B} / \overline{B^{\circ}}$, and by Fact $3.1(a)$ this action must be trivial. This shows that $[\bar{A}, \bar{B}] \leq \overline{B^{\circ}}$. Hence $[\bar{A}, \bar{B}] \leq \bar{A} \cap \overline{B^{\circ}}$, and since $\bar{A}$ and $\overline{B^{\circ}}$ commute we get that $[\bar{A}, \bar{B}] \leq Z(\bar{A})$. We may now apply Corollary 4.3 (with $H=\bar{A}$ and $X=\bar{B})$, and it gives that $[\bar{A}, \bar{B}]$ is a definably connected definable (abelian) subgroup of $Z(\bar{A})$ and furthermore that

$$
[\bar{A}, \bar{B}]=[\bar{A}, \bar{B}]_{\operatorname{dim}([\bar{A}, \bar{B}])} .
$$

Since $\left[A, B^{\circ}\right] \leq[A, B]$ and $[\bar{A}, \bar{B}]=[A, B] /\left[A, B^{\circ}\right]$ by lifting of commutators, the first point implies that $[A, B]$ is definable and definably connected. Now $\operatorname{dim}([A, B])=\operatorname{dim}([\bar{A}, \bar{B}])+\operatorname{dim}\left(\left[A, B^{\circ}\right]\right)$. If $A$ or $B^{\circ}$ is solvable, then as in Claim 6.2 we get that $[A, B]=[A, B]_{\operatorname{dim}([\bar{A}, \bar{B}])+\operatorname{dim}\left(\left[A, B^{\circ}\right]\right)}=[A, B]_{\operatorname{dim}([A, B])}$ with Lemma 2.1

In Corollary 6.5] we see from the proof that $[A, B] /\left[A, B^{\circ}\right]$ is abelian. In general it need not be trivial, or in other words it is not necessarily the case that $[A, B]=$ $\left[A, B^{\circ}\right]$. Consider for example the following semidirect product $B=A \rtimes\langle i\rangle$ where $A$ is a divisible abelian group and $i$ is an element of order 2 acting by inversion on $A$. One may of course realize a group definable in an o-minimal structure of this isomorphism type. Clearly $A=B^{\circ}$ is definably connected in such a group. We have $\left[A, B^{\circ}\right]=1$, but $[A, B]=A$.

Proof of Theorem 1.3. Since $A B$ is a definable subgroup of $G$, we may assume $G=A B$, with $A$ and $B$ two normal subgroups. All subgroups $[A, B],\left[A, B^{\circ}\right]$, and $\left[A^{\circ}, B\right]$ are normal in $A$ and $B$, and by Corollary 6.5 the two latter subgroups are 
definable and definably connected. By Fact 3.2 the normal definable subgroup $C:=\left[A^{\circ}, B\right]\left[A, B^{\circ}\right]$ of $[A, B]$ is definably connected.

By Corollary 6.5 we also have, as far as the commutator width is concerned, $\left[A^{\circ}, B\right]=\left[A^{\circ}, B\right]_{\operatorname{dim}\left(\left[A^{\circ}, B\right]\right)}$ if $A^{\circ}$ or $B^{\circ}$ is solvable. Working modulo $\left[A^{\circ}, B\right]$, we also find similarly as in Claim 6.2 or Corollary 6.5 (essentially by the additivity of the dimension, Lemma 2.1, and using Corollary 6.5 modulo $\left.\left[A^{\circ}, B\right]\right)$ that the commutator width of $C$ is bounded by $\operatorname{dim}(C)$ if $A^{\circ}$ or $B^{\circ}$ is solvable.

Working modulo $C$, we have $A^{\circ} \leq C_{A}(B)$ and $B^{\circ} \leq C_{B}(A)$, and Lemma 2.2 gives the finiteness of $[A, B]$ modulo $C$. In other words, $[A, B] / C$ is finite, and since $[A, B]$ is a finite extension of the definably connected definable subgroup $C$, we have $[A, B]$ definable, and $[A, B]^{\circ}=C$.

This completes our proof of Theorem 1.3 .

Remark 6.6. Clearly, by the last paragraph of the proof of Theorem 1.3 and Lemma 2.1, we also have that $[A, B]$ is of finite commutator width in Theorem 1.3 whenever $A^{\circ}$ or $B^{\circ}$ is solvable. We do not provide explicit bounds here, since they depend on the commutator width of the finite section $[A, B] /[A, B]^{\circ}$.

Applying inductively Theorem 1.3 we get the following corollary.

Corollary 6.7. Let $G$ be a group definable in an o-minimal structure such that $G^{\circ}$ satisfies the assumption $(*)$. Then, for each $n \geq 1, G^{n}$ and $G^{(n)}$ are definable and definably connected whenever $G$ is.

In view of Corollary [5.8. Corollaries 6.5 and 6.7 take the following specific form.

Corollary 6.8. Let $G$ be a group definable in an o-minimal structure such that $G^{\circ}$ is solvable. Then $\left[G^{\circ}, G\right]$ is definable and definably connected and, for each $n \geq 1$, $G^{n}$ and $G^{(n)}$ are definable and definably connected whenever $G$ is.

In principle, the commutator widths of the groups $\left[G^{\circ}, G\right],\left[G^{n}\right]^{\circ},\left[G^{(n)}\right]^{\circ}$, and all their definably connected variations are also controlled by their dimensions in Corollary 6.8, applying Theorem 1.3 ,

\section{FURTHER QUESTIONS AND REMARKS}

We finish the paper with some questions and remarks.

Question 7.1 (The normalization assumption). Can one prove versions of Theorem 6.1. Corollary 6.5, and Theorem 1.3, with $A$ normalizing $B$ only instead of $A$ and $B$ normalizing each other?

Question 7.2 (The commutator width). Let $G$ be a group definable in an ominimal structure.

(I) Assuming $G$ definably simple, is it the case that $[G, G]=[G, G]_{1}$ ?

(II) Can one get rid of the solvability assumption on $A$ or $B$ for the control of the commutator width of $[A, B]$ in Theorem 6.1]

(III) Assume $G$ satisfies the hypothesis of Theorem [1.3. Is there a uniform bound on the commutator width of the full group $[A, B]$, "modulo" the commutator width of $[A, B]^{\circ}$, as $A$ and $B$ vary as in that theorem? 
We strongly believe in a positive answer to question 7.2(I) (or at least in a very small commutator width). This corresponds to a conjecture of Ore in the case of finite simple groups (fully proved in that case in [8]). There are two ways to see a definably simple group as in Question 7.2 (I), either as the semialgebraic connected component of a real algebraic group or as (elementarily equivalent to) a simple Lie group (see [13]). When $G$ is definably compact, then it is known that every element is a commutator by an older theorem of Gotô about semisimple Lie groups (see 1]). Hence the question reduces to abstractly simple groups, as seen in the proof of Fact 5.3. In general the question remains open, but it is explicitly conjectured from the simple Lie groups point of view, that $G=[G, G]_{1}$ in [10, Conjecture A]. See also [1, page 15] for the same question and links with the Cartan decomposition.

Question 7.3. In a group definable in an o-minimal structure, let $H$ be a definable and definably connected subgroup and $X$ an arbitrary subset. When is it true that $[H, X]$ is definable and definably connected?

In the finite Morley rank context, Zilber's stabilizer argument yields a fully positive answer to the analog of Question 7.3 ([2, Corollary 5.29]). For groups in ominimal structures, Corollary 4.3 remains our most general answer to Question 7.3 . On the other hand, Question 7.3 may fail in that case even if $X$ is a normal subgroup of $H$, as the following example shows.

Example 7.4. Let $H$ be a definably compact definably connected definably simple nonabelian group definable in an o-minimal structure. Taking a sufficiently saturated model, we know that the smallest type definable subgroup $X=H^{\circ \circ}$ of bounded index in $H$ is normal, proper, and nontrivial [12. Then $[H, X]$ is not definable: otherwise, the proper normal definable subgroup $[H, X] \leq X<H$ would be trivial by definable simplicity of $H$, implying the existence of a nontrivial center in $H$.

We manage however to treat the case $H \subseteq X$ as a corollary of Theorem 6.1 as follows.

Corollary 7.5. Let $G$ be a group definable in an o-minimal structure, $H$ a definably connected definable subgroup which satisfies the assumption $(*)$, and $X$ any subset such that $H \subseteq X \subseteq N(H)$. Then $[H, X]$ is a definable and definably connected subgroup of $H$.

Proof. The group $H^{\prime}$ is definable and definably connected by Theorem 6.1. Since $H^{\prime}$ is normalized by $H$ and $X$, we may replace $G$ by $N\left(H^{\prime}\right)$, and working modulo $H^{\prime}$ we may assume $H$ to be abelian. Now, since $[H, X] \leq H=Z(H)$, Corollary 4.3 gives the definability and definable connectedness of $[H, X]$.

Of course, we can also obtain $[H, X]=[H, X]_{\operatorname{dim}([H, X])}$ as in the proof of Claim 6.4 when $H$ is solvable in Corollary 7.5 .

\section{ACKNOWLEDGEMENTS}

The authors thank Alessandro Berarducci, Gregory Cherlin, Ya'acov Peterzil, and Anand Pillay for various related conversations at Oberwolfach in January 2010. The authors also thank Dragomir Djokovic for hints concerning commutators in simple real Lie groups. 


\section{REFERENCES}

1. Armand Borel, Class functions, conjugacy classes and commutators in semisimple Lie groups, Algebraic groups and Lie groups, Austral. Math. Soc. Lect. Ser., vol. 9, Cambridge Univ. Press, Cambridge, 1997, pp. 1-19. MR.1635671 (99j:22020)

2. Alexandre Borovik and Ali Nesin, Groups of finite Morley rank, Oxford Logic Guides, vol. 26, The Clarendon Press, Oxford University Press, New York, 1994, Oxford Science Publications. MR 1321141 (96c:20004)

3. Annalisa Conversano, On the connections between groups definable in o-minimal structures and real Lie groups: the non-compact case, Ph.D. Thesis, University of Siena, 2009.

4. Adrien Deloro and Eric Jaligot, Groups of finite Morley rank with solvable local subgroups, To appear in Communications in Algebra, 2011.

5. Mário J. Edmundo, Solvable groups definable in o-minimal structures, J. Pure Appl. Algebra 185 (2003), no. 1-3, 103-145. MR2006422 (2004j:03048)

6. Ehud Hrushovski, Ya'acov Peterzil, and Anand Pillay, On central extensions and definably compact groups in o-minimal structures, J. Algebra 327 (2011), no. 1, 71-106. MR2746030

7. Otto H. Kegel and Bertram A. F. Wehrfritz, Locally finite groups, North-Holland Mathematical Library, Vol. 3, North-Holland Publishing Co., Amsterdam, 1973. MR0470081 (57:9848)

8. Martin W. Liebeck, E. A. O'Brien, Aner Shalev, and Pham Huu Tiep, The Ore conjecture, J. Eur. Math. Soc. (JEMS) 12 (2010), no. 4, 939-1008. MR2654085

9. Angus Macintyre, On $\omega_{1}$-categorical theories of abelian groups, Fund. Math. 70 (1971), no. 3, 253-270. MR0289280 (44:6471)

10. Dragomir Ž. Đoković, On commutators in real semisimple Lie groups, Osaka J. Math. 23 (1986), no. 1, 223-228. MR836712 (87g:22006)

11. Arkadi L. Onishchik and Èrnest. B. Vinberg, Lie groups and algebraic groups, Springer Series in Soviet Mathematics, Springer-Verlag, Berlin, 1990. Translated from the Russian and with a preface by D. A. Leites. MR1064110 (91g:22001)

12. Margarita Otero, A survey on groups definable in o-minimal structures, Model theory with applications to algebra and analysis. Vol. 2, London Math. Soc. Lecture Note Ser., vol. 350, Cambridge Univ. Press, Cambridge, 2008, pp. 177-206. MR2436142 (2010b:03042)

13. Ya'acov Peterzil, Anand Pillay, and Sergei Starchenko, Linear groups definable in o-minimal structures, J. Algebra 247 (2002), no. 1, 1-23. MR.1873380 (2002i:03043)

14. Derek J. S. Robinson, A course in the theory of groups, second ed., Graduate Texts in Mathematics, vol. 80, Springer-Verlag, New York, 1996. MR1357169 (96f:20001)

15. Maxwell Rosenlicht, On a result of Baer, Proc. Amer. Math. Soc. 13 (1962), 99-101. MR0147553(26:5068)

16. Lou van den Dries, Tame topology and o-minimal structures, London Mathematical Society Lecture Note Series, vol. 248, Cambridge University Press, Cambridge, 1998. MR 1633348 (99j:03001)

17. Frank O. Wagner, Stable groups, London Mathematical Society Lecture Note Series, vol. 240, Cambridge University Press, Cambridge, 1997. MR1473226 (99g:20010)

18. Robert B. Warfield, Jr., Nilpotent groups, Lecture Notes in Mathematics, Vol. 513, SpringerVerlag, Berlin, 1976. MR0409661 (53:13413)

19. Alex J. Wilkie, o-minimal structures, Astérisque (2009), no. 326, Exp. No. 985, vii, 131-142 (2010), Séminaire Bourbaki, Vol. 2007/2008. MR2605320

Departamento de Matemáticas, Universidad Autónoma de Madrid, 28049 Madrid, SPAIN

Current address: Universidad Complutense de Madrid, 28040 Madrid, Spain

Institut Fourier, CNRS, Université Grenoble I, 100 Rue des maths, BP 74, 38402 St Martin d'Hères Cedex, France

Departamento de Matemáticas, Universidad Autónoma de Madrid, 28049 Madrid, SPAIN 